CIENCIA Y SOCIEDAD

Volumen XXVII, Número 2

Abril - Junio 2002

\title{
ANÁLISIS DE UNA EVALUACIÓN TRANSCULTURAL DE LA INTELIGENCIA ACADÉMICA EN ALUMNOS DE SEXTO CURSO DE PRIMARIA
}

José Díaz Esteve*

RISIMEN

En este artículo el autor propone un modelo de procesamiento cognitivo para construir tests capaces de estimar la Inteligencia Académica (1.A.), entendiendo ésta como la capacidad de adquirır y utilizar eficazmente los conocimientos y destrezas aprendidas en el contexto adecuado. Este modelo permite agrupar los comportamientos seleccionados en cuatro clases de contenidos: semánticos, matemáticos, estructurales y conductuales y en cinco tipos de procesos mentales: conocimientos, destrezas, razonamientos, flexibilidad mental y capacidad de ponderación. Teniendo en cuenta este modelo el autor construido pruebas para evaluar la Inteligencia Académica en los diversos niveles escolares: Primaria, ESO, Bachillerato y Universidad. Este estudio se realiza con el test IAP3, que busca evaluar y contrastar los resultados en los contenidos curriculares básicos (Semánticos, Matemáticos. Espacio-estructurales y comportamientos) y procesos mentales fundamentales (Aprendizajes, Destrezas, Razonamiento, Flexibilidad y Capacidad ponderativa) desarrollados en cada sistema escolar, en dos muestras de alumnos de sexto curso de primaria de dos paises de habla hispana: España (137) y República Dominicana (121).

PALABRAS ClLAVES:

Modelo cognitivo de procesamiento, Inteligencia Académica, contenidos curriculares básicos, procesos mentales fundamentales.

\footnotetext{
: Iniversitat de València - España
} 
Desde principios del siglo XX se ha buscado definir el constructo Inteligencia, pero por su carácter excesivamente amplio se ha hecho casi imposible alcanzar este objetivo (Sánchez Cánovas y Sánchez López, 1994).

Estos intentos han estado dirigidos:

- por un lado a construir tests capaces de evaluar la Inteligencia, tarea que lleva implícita una definición operacional de la Inteligencia. Entre estos tests los más destacados son: el Binet y Simon (1905, 1908, 1911), el Stanford-Binet $(1916,1937,1961)$ de Terman, los Army Tests: Alpha y Beta (1917) y General Classification Test (1947), las Escalas de Weschler (WBI,1939; WBII,1941; WISC,1949; WAIS, 1955; WPPSI, 1967), los test Factor g de Catell "Culture Fair" (1971), los tests de Kaufman: el K-ABC- (para niños, 1983) y el KAIT (para adultos y adolescentes, 1993), el Detroit Test of Learning Aptitute-DTLA (1935), el DTLARevisado por Baker (1967), el DTLA-2 (1985) y DTLA-3 (1991) revisado por Hammil, el Differential Ability Scales -DAS- de Elliot (1990), Cognitive Assessment System -CAS- (1996).

- por otro lado, otro grupo de autores ha realizado una serie de trabajos destinados a investigar la naturaleza del constructo, tales como: Spearman (1904, 1907, 1910, 1913, 1927), Thorndike $(1907,1922,1927)$, Stern (1914), Thurstone $(1929,1935,1938,1941)$, Piaget (1955, 1972); Jensen (1969), Catell (1963, 1978), Horn (1968), Gardner (1993), Sternberg \& Alt. (1981, 1985 , 1988), Anderson (1993);

- finalmente otro grupo de autores se ha empeñado en delimitar el dominio de los comportamientos inteligentes, para luego clasificarlos y/o categorizarlos 
en diversos modelos teóricos, tales como: el cubo de Güilford (1965, 1966, 1967), el diagrama de Vernon (1971), la dicotomía (Int. fluida vs cristalizada) de Catell-Horn, las teorías jerarquizadas de Caroll (1993), de McGrew y Flanagan (1996). los esquemas de Anderson (1993), o el exaedro de Secadas (1995).

A pesar de estos intentos se ha comprobado que la magnitud del constructo es tal, que la tarea de definir la Inteligencia, parece un proceso inacabable, ya que la misma implicaría: observar y clasificar todos los comportamientos inteligentes, esquematizarlos, precisar y hacer verificaciones concurrentes, predecir a corto plazo, efectuar nuevas precisiones y predicciones, formular hipótesis plausibles, y, por último, desarrollar modelos teóricos capaces de integrar los viejos y los nuevos conocimientos y datos.

\section{REVISIÓN HISTÓRICA DEL CONSTRUCTO}

En las primeras décadas del s.XX se pensaba en la unicidad del constructo. Pero, luego, desde los finales de la década de los 20 y sobre todo en la de los 30, algunos autores, (Scheringer, 1928; Scudder \& Raubenheimer, 1931; Broom,1931; Alexander, 1937, Weschler, 1939, ...) comienzan a hacer una clara distinción entre dos facetas de una misma Inteligencia: la Teórica (o académica, o verbal) y la Práctica (o manipulativa, o mecánica, o social). En las décadas posteriores $(40,50,60,70, \ldots)$ se realizan, en diversos niveles educativos y ambientes culturales, diversos estudios correlacionales entre la evaluación de estos aspectos de la Inteligencia y el rendimiento académico y/o laboral (Williamson \& Darley, 1935; Gough, 1948; Singh-Baldev, 1956; Tsudsuki, 1957; Shuluke, 1958; Carter, 1959; Sechrest \& Jackson, 1961; McBee \& Duke, 1961; Kolowinsky, 1961; Yamamoto, 1964; Guilford, Hoepfner \& Petersen, 1965; Warhadpande \& Kuller, 1966; Keating, 1978). 
En los años 80 y 90 aparece un grupo de autores, tales como: Sternberg y colaboradores $(1981,1985,1989)$ Ford \& Tisak (1983), Wagner (1988), Stewart (1998) que prefieren hablar de varias clases o tipos de inteligencia que perciben bien diferenciadas entre sí, estas son: la práctica o sentido común (estructurada sobre los llamados conocimientos tácitos), la social (habilidad para adaptarse a las diversas situaciones sociales), la emocional (capacidad para identificar y manejar adecuadamente las emociones) y la académica (capacidad de adquirir y utilizar adecuadamente los conocimientos en el contexto adecuado, lo que implica percibir y asumir la responsabilidad de asumir las limitaciones y la capacidad de trasladar el pensamiento a la acción). En estos años aparencen, también, varios trabajos que comparan y contrastan entre sí, estos tipos de inteligencia: la práctica vs la académica (Wagner \& Alt., 1990, 1994; Torf \& Sternberg, 1998; Sternberg \& Alt., 1996, 2000), la social vs la académica (Riggio \& alt, 1991; Wong \& Alt., 1995; Jones \& Day, 1996, 1997).

\section{LA INTELIGENCIA ACADÉMICA}

Dentro de la inteligencia académica, considerada como la capacidad para solucionar problemas académicos (Jones \& Day, 1996), se han distinguido a su vez dos facetas (Allik \& Realo, 1997; Lee, Wong \& Alt, 2000): la fluida (el conjunto de habilidades inherentes que facilitan el aprendizaje al sujeto ) y la cristalizada, (el conjunto de conocimientos implícitos y destrezas/ automatismos que el sujeto ha adquirido y desarrollado a través de los diversos procesos enseñanza/aprendizaje). Al parecer, de acuerdo Sternberg y Wagner, la inteligencia académica podría ser identificada con el tradicional factor $g$, aunque envuelto en otro lenguaje ( $g$-océntrico), identificación que es criticada, severamente, por Jensen (1993) y que es claramente diferenciable del rendimiento académico, expresado por cualquiera de estos valores: las notas o calificaciones obtenidas, el índice académico, la 
motivación para aprender, el nivel educacional alcanzado por el sujeto, los premios o distinciones recibidos, ... (Oliver, R.N, 1994).

Esta patente dificultad para delimitar y clasificar el dominio de comportamientos intelectivos ha llevado a muchos constructores de tests a olvidarse de buscar una definición sustantiva de la inteligencia y pasar a ofrecer una definición operacional, fijándose sólo en algunos aspectos funcionales de la actividad mental, que permite crear tests adecuados a las necesidades o propósitos de la investigación. En ese sentido se ha dicho que la "inteligencia es aquello que cada autor de test, dice que es".

Así, pues, el autor siguiendo las directrices de Sternberg y Wagner, intenta evaluar algunos aspectos de la inteligencia académica, definiendola:

- descriptivamente como "el nivel de desarrollo mental que alcanza un alumno en un determinado nivel escolar, a consecuencia del proceso enseñanza/aprendizaje académico"

- y operacionalmente como "la capacidad para:

a. captar, asimilar y reconocer objetos y/o conceptos expresados mediante palabras, símbolos numéricos, figuras o comportamientos,

b. operar con estos contenidos de diversas maneras, tales como: recordar, comprender, clasificar, ordenar, completar, agrupar en conceptos superiores, contraponer y emitir juicios, elaborar nuevos,

c. razonar, resolver problemas, cambiar, reproducir, establecer relaciones,

d. comprender, profundizar e inferir consecuencias de los mismos,

e. actuar con propósito y/o anticiparse a situaciones y operar eficazmente en el medio ambiente (Díaz, 2000, pág 279). 


\section{MODELO COGNITIVO DE PROCESAMIENTO}

Definido el atributo, en este caso la inteligencia académica, se ha hecho necesario elaborar un modelo de procesamiento cognitivo (model of cognitive funtions- Das, 1988), en el que se señalan las los conetenidos y funciones sobre los cuales se va a construir el test. Para elaborar este modelo se ha procedido a realizar una amplia revisión de los comportamientos mentales más utilizados para evaluar la inteligencia en los principales tests desarrollados hasta el presente, reunirlos todos en una especie de mapa conceptual, para comenzar, luego, a: seleccionar aquellos, que en opinión del autor y equipo podían estar más relacionados con la inteligencia académica. Seleccionados estos comportamientos se diferenciaron y organizaron entre sí para constituir una especie de modelo metodológico jerárquico, que viene expresado en las figura 1.

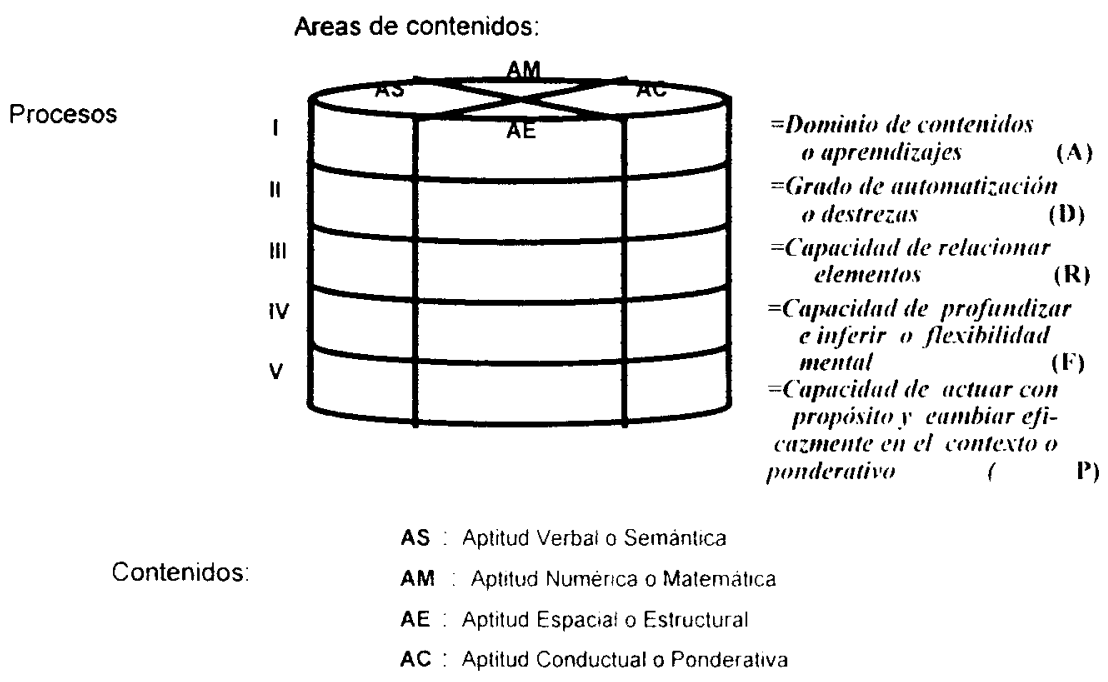

FIGURA N. ${ }^{\circ} 1$

Modelo de procesamiento ligado a la definición de inteligencia académica adoptada 
De modo, que de acuerdo a este modo de definir la inteligencia, se puede afirmar que en el hombre existe una capacidad general para operar mentalmente, siendo ésta como una estructura interna, que permite amoldarse a diversas clases de contenidos. Estos contenidos que han sido clasificados en cuatro categorías:

- palabras (AS: aptitud semántica),

- números o contenidos matemáticos (AM: aptitud matemática),

- figuras o estructuras espaciales (AE: aptitud espacioestructural) y

- conductas o estimaciones ponderativas (AC: aptitud conductual o ponderativa).

Estas categorías generan, a su vez, cuatro tipos de Inteligencia, que han sido considerados importantes, dentro del contexto académico escolar, para apreciar en primer lugar el nivel de desarrollo de las funciones mentales que ha alcanzado cada sujeto en un momento educativo dado (evaluación estática), así como los cambios sucedidos en los mismos después de aplicar un proceso educativo normal o terapéutico mediante una intervención psicoeducativa intensiva (evaluación dinámica). Una decripción de los contenidos y procesos seleccionados para ser evaluados puede ser vista en las referencias bibliográficas citadas (Díaz 2001a y Díaz 2001b).

Definido el modelo de procesamiento y el modelo de medición (items de selección múltiple con cuatro alternativas) se procedió a redactar un copioso banco de items ayudado por los alumnos de Practicum en Psicología Evaluativa y Educativa de la Facultad de Psicología de la Universidad de Valencia, durante varios cursos académicos (1995-2000). Estos items fueron administrados a grupos pequeños (30-50 alumnos) en estos niveles educativos: 
- Primaria.1 (2, 3, 4 cursos),

- Primaria.2 (4, 5, 6 cursos),

- ESO (Enseñanza Secundaria Obligatoria),

- Bachillerato y

- Universidad.

Los resultados de los análisis psicométricos fueron guardados en un banco de items a través del programa LXR. Test (5.1) llegando a disponer de unos 700 items. Este banco finalmente se ha dividido en otros cuatro:

Intel P.2 items experimentados con alumnos del $2^{\circ}$ nivel de primaria,

Intel P.3 items experimentados con alumnos del $3^{\circ}$ nivel de primaria,

Intel ESO items experimentados en alumnos de ESO,

Intel BUP items experimentados con alumnos de Bachillerato,

Intel $U \quad$ items experimentados con alumnos universitarios.

El programa LXR.Test 5.1 (1995), permite elaborar diversos formas del test en cada uno de los niveles educativos señalados, de acuerdo a las necesidades y aspectos intelectuales que se quieran preferentemente medir.

En este artículo se presenta un estudio comparativo transcultural entre los resultados de la aplicación del IAP.3, Forma A (tercer nivel 5-6 de primaria) a dos muestras de alumnos de sexto curso. 


\section{Descripción de la muestra}

Dado que el objetivo de la investigación era efectuar una evaluación transcultural de la inteligencia académica del escolar se buscaron dos muestras: una formada por 135 alumnos (M1) que durante el curso escolar 2000-01 cursaban el sexto curso de primaria en la provincia de Valencia (España) y otra formado por 121 alumnos (M2) de Santo Domingo (República Dominicana), que así mismo estaban cursando el sexto curso de primaria en tres centros privados de la capital del País. En la Tabla N. ${ }^{\circ} 1$ se presenta la distribución de las dos muestras por sexo y edad:

TABLA N. ${ }^{\circ} 1$

La Distribución de las Muestras por Sexo y Edad

\begin{tabular}{|c|c|c|c|c|c|c|c|c|}
\hline \multirow{3}{*}{ Sexo } & \multicolumn{4}{|c|}{ M1 (española) } & \multicolumn{4}{|c|}{ M2 (dominicana) } \\
\hline & $\mathrm{H}$ & & & $T$ & & & $M$ & $T$ \\
\hline & 68 & & & 135 & & & 61 & 121 \\
\hline \multirow[t]{2}{*}{ Edad } & 10 & 11 & 12 & 13 & 10 & 11 & 12 & 13 \\
\hline & 2 & 112 & 21 & - & 4 & 86 & 30 & 1 \\
\hline
\end{tabular}

La prueba fue aplicada de forma colectiva en horario de clase, la primera (M1) por estudiantes del II clico de Psicopedagogía como parte de una práctica del módulo "Introducción a los Métodos de Investigación y Medición Psicológicos: construcción de cuestionarios, escalas y tests" ( 6 créditos), que el autor impartió en el I Semestre del curso académico 2000-01 en la Universitat de València, y la segunda muestra (M2) por el propio autor, con la ayuda del psicologo escolar de cada centro, aprovechando la visita que el autor hizo para impartir un curso en una universidad dominicana. 


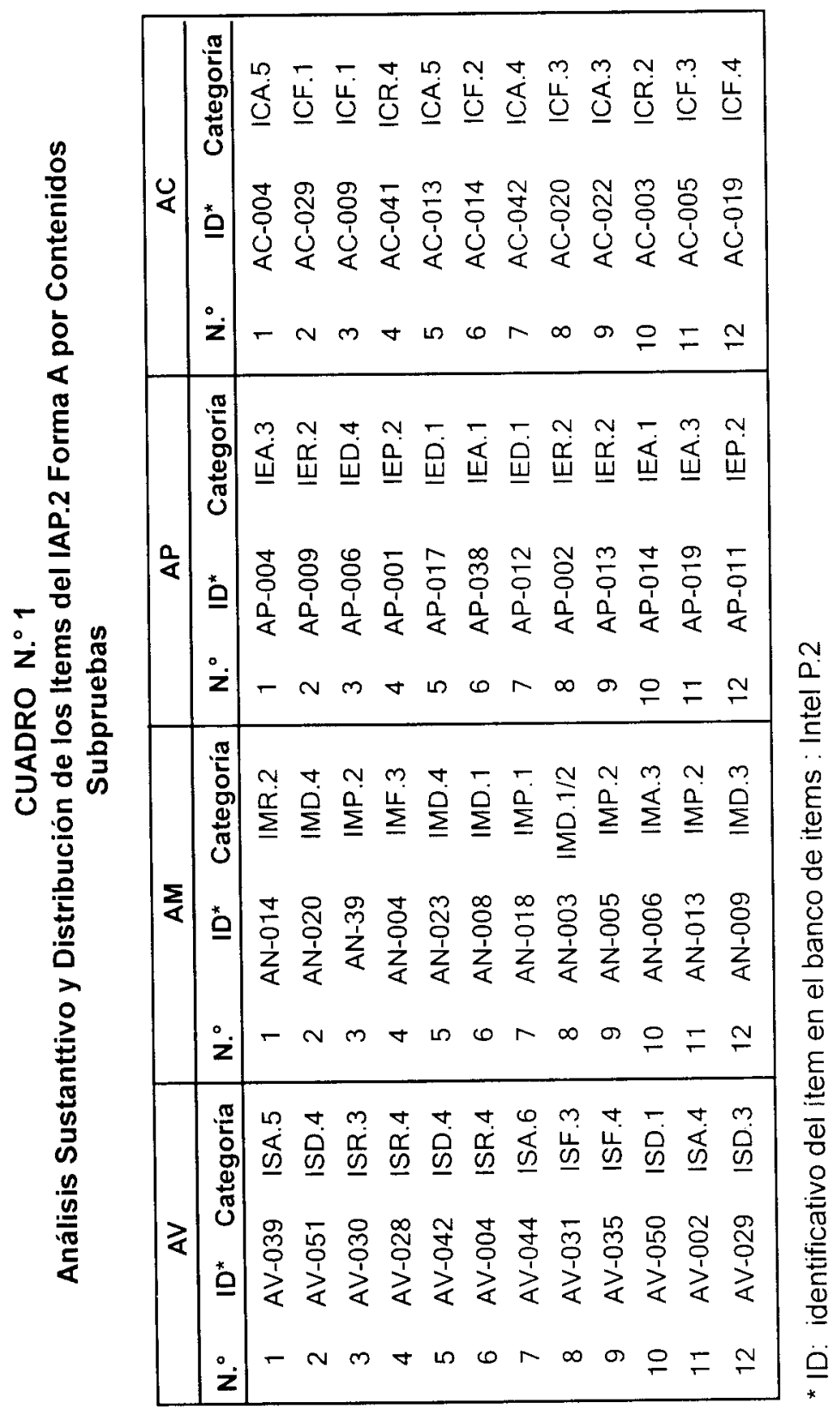




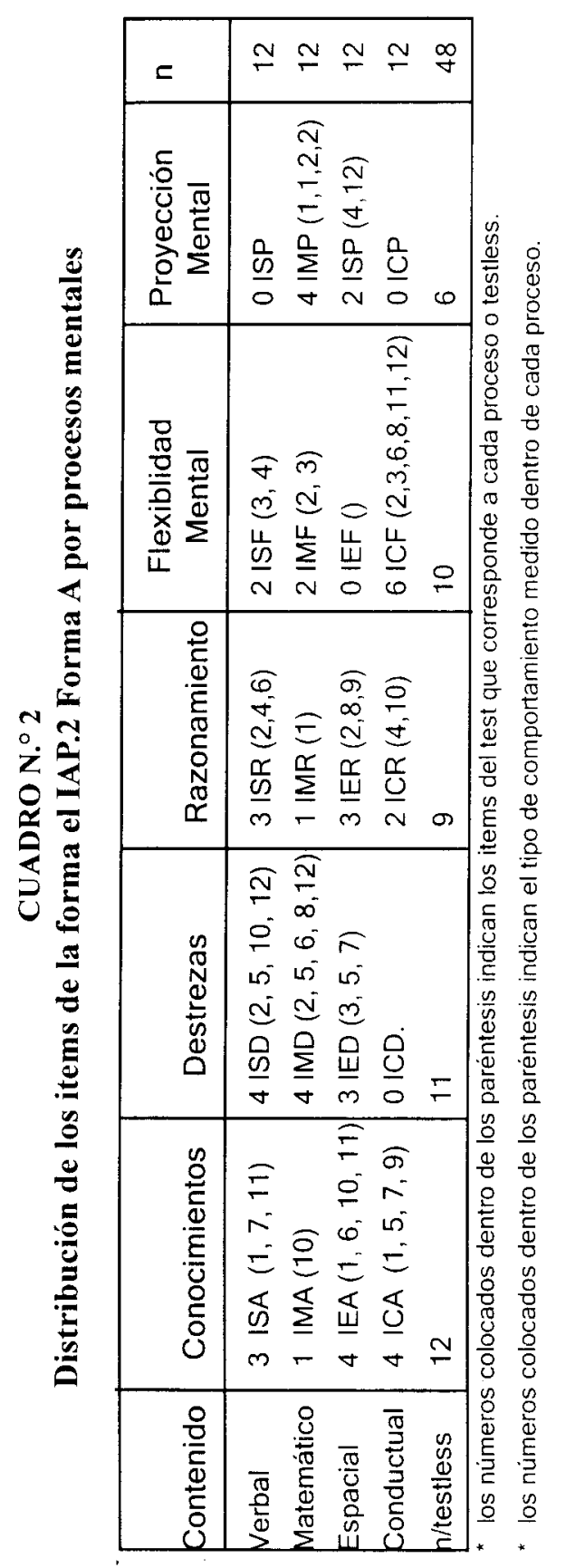

274 


\section{Instrumento de medida}

El test IAP.3 Forma A (Test de Inteligencia Académica aplicable al $3^{\circ}$ nivel de Primaria, 4-6 curso) forma parte de un conjunto de tests desarrollados para evaluar las funciones mentales más ligadas al rendimiento académico en los diversos niveles escolares.(IAP2, IAP3, IAE, IAB, IAU). El IAP.3 Forma A está formado por cuatro subpruebas con doce elementos en cada una de ellas (Cuadro N. ${ }^{\circ} 1$ )

En el Cuadro $N .^{\circ} 2$ se resume el número de items que tiene cada una de las cinco procesos o funciones mentales, que permite estimar el modelo de procesaminento adoptado.

Este tipo de pruebas, se puede construir, como se ha indicado antes, de acuerdo a las necesidades evaluativas del constructor o del centro sobre cualquiera de los bancos de items citados, cuyos items están ya calibrados. En la presente investigación, dado el caso que se trataba de dos muestras de poblaciones cultural y educativamente distintas, aunque hablan el mismo idioma, algunos items tuvieron que ser modificados o sustituídos, pero procurando mantener los mismos tipos de items y con características psicométricas de los items originales.

\section{Variables controladas}

Dado que el objetivo básico de esta investigación fue realizar un estudio comparativo entre las dos muestras señaladas a través del IAP.2, se han establecido:

Las siguientes variables independientes:

$\begin{array}{ll}\text { Sexo: } & \mathbf{1}-\text { hombre; } \\ & \mathbf{2}-\text { mujer. } \\ \text { Edad: } & \mathbf{1}-10 \text { años; } \\ & \mathbf{2}-11 \text { años; } \\ & \mathbf{3}-12 \text { años. }\end{array}$


Centro: donde estudian los sujetos. De acuerdo al tipo de muestra se han estimado estas variables:

- en la muestra española

1. centros situados en Valencia

2. centros situados en ciudades de la provincia

(En la muestra española no se tuvo en cuenta la variable: privado o público, en el supuesto que no debe haber diferencia entre ambas categorías).

- en cambio en la muestra dominicana se ha estimado esta variable de acuerdo a localización de los centros:

1. Zona residencial antigua (Gascue)

2. Zona de crecimiento más reciente (Independencia-Feria-Cancillería)

3. Zona residencial más moderna ( $\mathrm{Naco}$ )

Los tres centros son privados, a ellos asisten alumnos de clase media alta.

País: 1 - muestra española;

2 - muestra dominicana

Y las siguientes variables dependientes, correspondientes a los cuatro componentes del test y una quinta la Total:
AV (aptitud verbal)
= suma de items acertados en la $1^{\text {a }}$ prueba
AM (aptitud matemática) = suma de items acertados en la $2^{\mathrm{a}}$ prueba
AE (aptitud espacial) = suma de items acertados en la $3^{\mathrm{a}}$ prueba 
AC (aptitud ponderativa) = suma de items acertados en la $4^{\mathrm{a}}$ prueba

Total = suma de items acertados en la cuatro pruebas.

\section{Procedimiento}

Las respuestas de los sujetos fueron procesadas, para cada una de las muestras, a través del programa ITEMAN para windows del Microcart Testing System (1995). Los" outps" obtenidos informan acerca: de los estadísticos de los items, de los estadisticos del test y de las puntuaciones de los sujetos en cada una de las subpruebas. Con estos resultados se procedió, en primer lugar a buscar, dentro de cada muestra, las posibles diferencias entre las puntuaciones obtenidas en cada una de la subpruebas del test en los distintos valores de las variables independientes: Sexo, Edad y Centro, y en segundo lugar a comparar las puntuaciones del test a través de los valores de la variable país para ver las posibles diferencias en las aptitudes evaluadas. Para lograr estos objetivos se procesaron los datos a través de un ANOVA factorial utilizando la rutina del paquete estadístico (StatView.II, 1990).

Finalmente se han querido ver las posibles diferencias entre el nivel de desarrollo de las funciones mentales básicas así como descubrir las posibles deficiencias en la Inteligencia Académica, desarrolladas en las dos muestras como consecuencia de los procesos enseñanza-aprendizaje del sistema educativo que cada país ha adoptado. Para señalar estos aspectos se ha procedido a realizar un análisis diferencial del funcionamiento de los items utilizando dos métodos: uno estadístico y otro psicométrico, como luego se verá.

\section{Resultados}

Los resultados obtenidos en cada uno de los cálculos señalados se expresan en las siguientes tablas para cada variable independiente controlada: 
$1^{\circ}$ Sexo (1 Hombres, 2 Mujeres) en cada una de las muestras estudiadas (M1 Española, M2 Dominicana, M3 Total)

TABLA N. ${ }^{\circ} 2$

Resultados del Anova de las Puntuaciones en el Tests por Sexos y Muestras

\begin{tabular}{|c||c|c||c|c|c|c|}
\hline Test & $\mathbf{F}(\mathrm{M} 1)$ & $\mathbf{p}$ & $\mathbf{F}(\mathrm{M} 2)$ & $\mathbf{p}$ & $\mathbf{F}(\mathrm{M} 3)$ & $\mathbf{p}$ \\
\hline AV & 2.975 & 0.086 & 0.243 & 0.632 & 3.270 & 0.071 \\
AM & 0.002 & 0.964 & 0.556 & 0.457 & 0.066 & 0.795 \\
AE & 0.684 & 0.406 & 0.043 & 0.838 & 1.169 & 0.280 \\
AC & 1.031 & 0.315 & 0.759 & 0.838 & 0.839 & 0.360 \\
Total & 1.228 & 0.269 & 0.645 & 0.623 & 2.990 & 0.156 \\
\hline
\end{tabular}

* Existe diferencia significativa entre, medias, $(p<0.05)$

$2^{\circ}$ Edad $(\mathbf{1 0}, \mathbf{1 1}, \mathbf{1 2})$ en cada una de las muestras estudiadas (M1 Española, M2 Dominicana, M3 Total)

TABLA N.$^{\circ} 3$

Resultados del ANOVA de las Puntuaciones en el Test por Edades y Muestras

\begin{tabular}{|l||l|l|l|l|l|l|}
\hline Test & $\mathbf{F}(\mathrm{M} 1)$ & $\mathbf{p}$ & $\mathbf{F}(\mathrm{M} 2)$ & $\mathbf{p}$ & $\mathbf{F}(\mathrm{M} 3)$ & $\mathbf{p}$ \\
\hline AV & 0.581 & 0.560 & 2.975 & 0.086 & 3.963 & $0.020^{*}$ \\
$\mathrm{AM}$ & 3.271 & $0.041^{\star}$ & 0.002 & 0.964 & 1.999 & 0.137 \\
$\mathrm{AE}$ & 1.093 & 0.339 & 0.684 & 0.409 & 1.856 & 0.158 \\
$\mathrm{AC}$ & 1.064 & 0.348 & 1.031 & 0.311 & 1.874 & 0.443 \\
\hline Total & 2.065 & 0.122 & 1.645 & 0.269 & 1.623 & 0.199 \\
\hline
\end{tabular}

existe diferencia significativa entre medias, $(p<0.05)$. (lute se manifiesta en el post análisis entre grupo 2 (11 años) y 3 (12 años) 
$3{ }^{\circ} \mathrm{P}$ aís $(1,2)$ en ambas muestras divididas en dos categorías (M1 España, M2 República Dominicana)

TABLA N.$^{\circ} 4$

Resultados del ANOVA de las Puntuaciones del Test por Países

\begin{tabular}{|c||c|c||c|c|c|}
\hline Test & $\mathbf{F}$ & $\mathbf{p}$ & $\mathbf{M}$ 1 & M 2 & Dif \\
\hline AV & 0.724 & 0.395 & 6.686 & 6.883 & -0.197 \\
AM & 6.375 & $0.012^{*}$ & 6.066 & 6.737 & -0.671 \\
AE & 17.613 & $0.0001^{*}$ & 6.380 & 7.504 & -1.123 \\
AC & 22.978 & $0.0001^{*}$ & 8.686 & 7.511 & 1.175 \\
\hline Total & 1.298 & 0.2556 & 27.818 & 28.635 & -0.817 \\
\hline
\end{tabular}

(*) Existe diferencia significativa entre medias, $(p<0.05)$

$4^{\circ}$ Localización del centro: esta variable se ha estudiado en cada una de las categorías de la variable país por separado, ya que esta toma valores distintos en cada una de las muestras. Así:

- en la muestra española presenta estos valores, centros situados:

1. en Valencia Capital;

2. fuera Valencia Capital;

- en la muestra dominicana, en cambio, al estar situados todos los centros en la capital, la variable se ha dividido de acuerdo a las zonas donde están situados los centros, distinguiéndose, pues, tres zonas:

1. Zona Matahambre (zona donde abundan edificios públicos),

2. Zona Gazcue (zona de vivienda antigua de la ciudad),

3. Zona Naco (zona comercial y de vivienda más moderna de buen nivel). 
Los resultados de estos análisis se pueden ver en las Tablas N. ${ }^{\circ} 5$ y N. ${ }^{\circ} 6$

TABLA N. ${ }^{\circ} 5$

Resultados del ANOVA de las Puntuaciones en el Test por Situación del Centro en la Muestra Española

\begin{tabular}{|c|c|c|}
\hline Test & $\mathrm{F}$ & $\mathrm{p}$ \\
\hline AV & 1.926 & 0.167 \\
AM & 1.430 & 0.233 \\
AE & 3.642 & 0.067 \\
AC & 0.799 & 0.388 \\
\hline Total & 3.771 & 0.077 \\
\hline
\end{tabular}

(") Existe diferencia significativa entre medias. $(p<0.05)$

TABLA N. ${ }^{\circ} 6$

Resultados del ANOVA de las Puntuaciones en el Test por Situación del Centro en la Muestra Dominicana

\begin{tabular}{|c|c|l|}
\hline Test & $\mathbf{F}$ & \multicolumn{1}{|c|}{$\mathbf{p}$} \\
\hline AV & 3.760 & $0.026^{\star}$ \\
AM & 2.506 & 0.086 \\
AE & 3.389 & $0.037^{\star}$ \\
AC & 4.763 & $0.013^{\star}$ \\
\hline Total & 7.821 & $0.0006^{*}$ \\
\hline
\end{tabular}

(*) Existe diferencia significativa entre medias. $(p<0.05)$

Las diferencias se manifiestan, en el caso que las haya, en el post-análisis, al comparar la localización de los centros entre sí, como se puede observar en las Tablas $\mathrm{N} .^{\circ} 7$ y N. ${ }^{\circ} 8$ : 


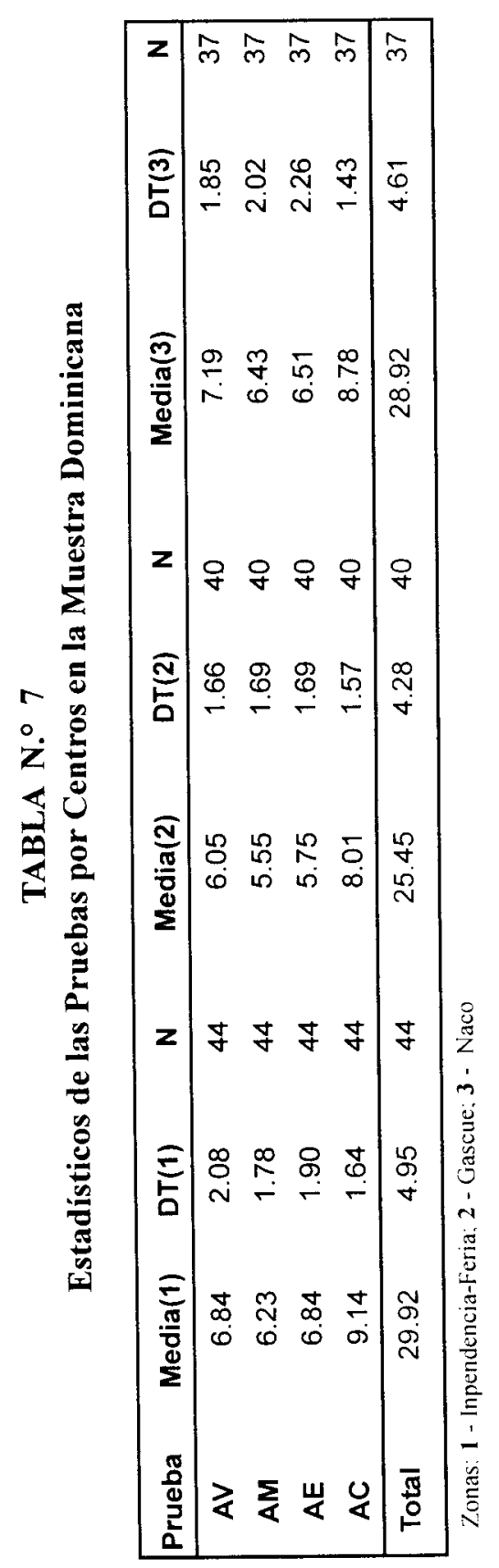


TABLA ${ }^{\circ}{ }^{\circ} 8$

Diferencias Aparecidas en el Post-análisis en la Muestra

Dominicana

\begin{tabular}{|c|c|c|c|}
\hline \multirow{2}{*}{ Test } & Grupos & Dif Medias & Scheffe F \\
\hline AV & $2-3$ & \multicolumn{3}{|c|}{-1.939} & $3.525^{\star}$ \\
\cline { 2 - 4 } AM & \multicolumn{3}{|c|}{ no aparecen diferencias entre grupos } \\
\cline { 2 - 4 } AE & $1-2$ & 1.09 & $3.865^{\star}$ \\
AC & $1-2$ & 1.036 & $4.658^{\star}$ \\
\hline Total & $1-2$ & 3.595 & $6.315^{\star}$ \\
& $2-3$ & -3.409 & $5.999^{*}$ \\
\hline
\end{tabular}

(9) existe diferencia significativa entre medias, $(\mathrm{p}<0.05)$

\section{Análisis diferencial del funcionamiento de los items}

Dado que uno de los objetivos básicos de esta investigación era realizar un estudio comparativo entre las muestras de los dos paises, buscando las posibles diferencias entre el nivel de desarrollo de las funciones mentales básicas, así como, descubrir las posibles deficiencias en la Inteligencia Académica desarrolladas en las dos muestras como consecuencia de los procesos enseñanza-aprendizaje del sistema educativo que cada país ha adoptado, se ha decidido añadir a los resultados anteriores un análisis diferencial del funcionamiento de los items, que permita ofrecer alguna señal de las propiedades estadísticas de los items entre los grupos. Para efectuar este análisis se han utilizado dos procedimientos: uno meramente estadístico y otro de carácter más psicométrico.

\section{$1^{\circ}$ Resultados del procedimiento estadístico}

El procedimiento estadístico se ha efectuado comparando y contrastando la proporción de aciertos entre las dos muestras en cada uno de los items, mediante el estadístico $Z$ para proporciones y cuyos resultados se exponen en la tabla 5: 
TABLA N. 5

Comparación de Proporciones de Acierto en las Dos Muestras

\begin{tabular}{|c|c|c|c|c|}
\hline Subtest & & $\begin{array}{l}\text { Muestra } \\
\text { Española }\end{array}$ & $\begin{array}{c}\text { Muestra } \\
\text { Dominicana }\end{array}$ & $\begin{array}{l}\text { Estadístico } \\
\text { de contraste }\end{array}$ \\
\hline \multirow[t]{13}{*}{$\mathbf{A S}$} & ítem & pi & pi & $\mathbf{Z}$ \\
\hline & 01 & 0.85 & 0.93 & $-2.09^{\star}$ \\
\hline & 02 & 0.50 & 0.49 & 0.16 \\
\hline & 03 & 0.91 & 0.93 & -0.59 \\
\hline & 04 & 0.56 & 0.54 & 0.32 \\
\hline & 05 & 0.18 & 0.34 & $-2.95^{\star}$ \\
\hline & 06 & 0.63 & 0.43 & $3.28^{\star}$ \\
\hline & 07 & 0.87 & 0.88 & -0.24 \\
\hline & 08 & 0.30 & 0.12 & $3.67^{\star}$ \\
\hline & 09 & 0.69 & 0.66 & 0.51 \\
\hline & 10 & 0.41 & 0.31 & 1.68 \\
\hline & $11 \#$ & 0.28 & 0.82 & $-10.41^{\star}$ \\
\hline & 12 & 0.72 & 0.26 & $8.31^{\star}$ \\
\hline \multirow[t]{13}{*}{$\mathbf{A M}$} & ítem & pi & pi & $\mathbf{Z}$ \\
\hline & 01 & 0.70 & 0.76 & -1.09 \\
\hline & 02 & 0.88 & 0.91 & -0.79 \\
\hline & 03 & 0.72 & 0.74 & -0.36 \\
\hline & 04 & 0.47 & 0.21 & $4.60^{\star}$ \\
\hline & $05^{*}$ & 0.48 & 0.58 & -1.61 \\
\hline & 06 & 0.91 & 0.84 & 1.69 \\
\hline & 07 & 0.45 & 0.59 & $-2.27^{\star}$ \\
\hline & 08 & 0.31 & 0.12 & $3.85^{*}$ \\
\hline & 09 & 0.53 & 0.40 & $2.10^{\star}$ \\
\hline & 10 & 0.50 & 0.27 & 3.91 \\
\hline & 11 & 0.58 & 0.51 & 1.13 \\
\hline & 12 & 0.21 & 0.12 & $1.97^{\star}$ \\
\hline
\end{tabular}




\begin{tabular}{|c|c|c|c|c|}
\hline \multicolumn{2}{|l|}{ Subtest } & \multirow{2}{*}{$\begin{array}{c}\begin{array}{c}\text { Muestra } \\
\text { Española }\end{array} \\
\text { pi } \\
\end{array}$} & \multirow{2}{*}{$\begin{array}{c}\begin{array}{c}\text { Muestra } \\
\text { Dominicana }\end{array} \\
\text { pi }\end{array}$} & \multirow{2}{*}{$\begin{array}{c}\begin{array}{c}\text { Estadístico } \\
\text { de contraste }\end{array} \\
\mathrm{Z}\end{array}$} \\
\hline $\mathbf{A E}$ & ítem & & & \\
\hline & 01 & 0.90 & 0.87 & 0.75 \\
\hline & 02 & 0.61 & 0.39 & $3.62^{\star}$ \\
\hline & 03 & 0.68 & 0.60 & 1.33 \\
\hline & 04 & 0.70 & 0.60 & 1.68 \\
\hline & 05 & 0.18 & 0.08 & $2.44^{\star}$ \\
\hline & 06 & 0.72 & 0.67 & 0.87 \\
\hline & 07 & 0.56 & 0.35 & $3.46^{*}$ \\
\hline & 08 & 0.64 & 0.48 & $2.61^{*}$ \\
\hline & 09 & 0.64 & 0.50 & $2.24^{\star}$ \\
\hline & $10^{*}$ & 0.79 & 0.65 & $2.51^{\star}$ \\
\hline & 11 & 0.74 & 0.83 & -1.77 \\
\hline & 12 & 0.37 & 0.37 & 0.00 \\
\hline \multirow[t]{13}{*}{$\mathrm{AC}$} & ítem & pi & pi & $Z$ \\
\hline & $01^{*}$ & 0.59 & 0.85 & $-4.89^{\star}$ \\
\hline & 02 & 0.92 & 0.97 & -0.98 \\
\hline & 03 & 0.88 & 0.94 & -1.70 \\
\hline & 04 & 0.69 & 0.93 & $-5.23^{\star}$ \\
\hline & 05 & 0.88 & 0.58 & $5.68^{*}$ \\
\hline & $06^{\#}$ & 0.65 & 0.97 & $-3.82^{\star}$ \\
\hline & 07 & 0.53 & 0.55 & -0.32 \\
\hline & 08 & 0.49 & 0.55 & -0.96 \\
\hline & $09^{*}$ & 0.73 & 0.96 & $-5.48^{\star}$ \\
\hline & 10 & 0.58 & 0.45 & 2.10 \\
\hline & 11 & 0.36 & 0.30 & 1.02 \\
\hline & $12^{*}$ & 0.23 & 0.64 & $-7.44^{*}$ \\
\hline
\end{tabular}

+ cuando $Z>1.96$ aparece diferencia significativa a dos colas entre las proporciones al $5 \%$

cuando $\mathbf{Z}>\mathbf{2 . 5 8}$ aparece diferencia significativa a dos colas entre las proporciones al $1 \%$

item con valores paramétricos similares. que han sido modificados por tener contenidos diferenciables culturalmente 
Este procedimiento, indica que en 23 de los 48 items aparecen diferencias significativas $(*)$. Esta diferencia permite inducir solamente, que la probabilidad de responder correctamente es mayor para un grupo que para otro, pero no que dicha diferencia sea debida a que en este grupo el nivel de habilidad sea mayor que en el otro, ya que la diferencia pudo estar afectada por la composición de los grupos. Esta diferencia es identificada como Impacto (Ackerman, 1992)

\section{$2^{\circ}$ Resultados del Procedimiento Psicométrico}

El procedimiento psicométrico, se efectuó utilizando el DIF, que permite señalar que la probabilidad de responder correctamente al ítem está ligada mayormente al nivel de aptitud de grupo. Se han señalado diversos procedimientos para detectar el DIF. En este trabajo, por su sencillez, bajo costo computacional y buenos resultados se ha utilizado el MHDIF de Fidalgo (1994) que está fundamentado en el procedimiento Mantel-Hanszel y permite calcular el $\mathrm{MH}-\mathrm{CHI} 2$ (ji cuadrado $\mathrm{MH}$ ), que señala claramente la presencia o ausencia de DIF, observando la columna DIF estos criterios: $0=$ ausencia de DIF, $1=$ presencia DIF. $\mathrm{Si}$ además se observan los signos de la columna MH D-DIF, se podrá apreciar el sentido del DIF; así: + cuando el DIF es a favor del Grupo Focal (muestra española), y - cuando esta a favor del Grupo de Referencia (muestra dominicana), como puede observarse en la Tabla N. ${ }^{\circ} 6$

Los datos de las Tablas $\mathrm{N} .{ }^{\circ} 5 \mathrm{y} \mathrm{N} .^{\circ} 6$ han sido esquematizados en el Cuadro N. ${ }^{\circ}$, en el que se aprecian tres fuentes de variabilidad: el impacto (I), el DIF (D) y los modificaciones (M) hechas a los items por estar posiblemente influenciados de contenidos culturales. 


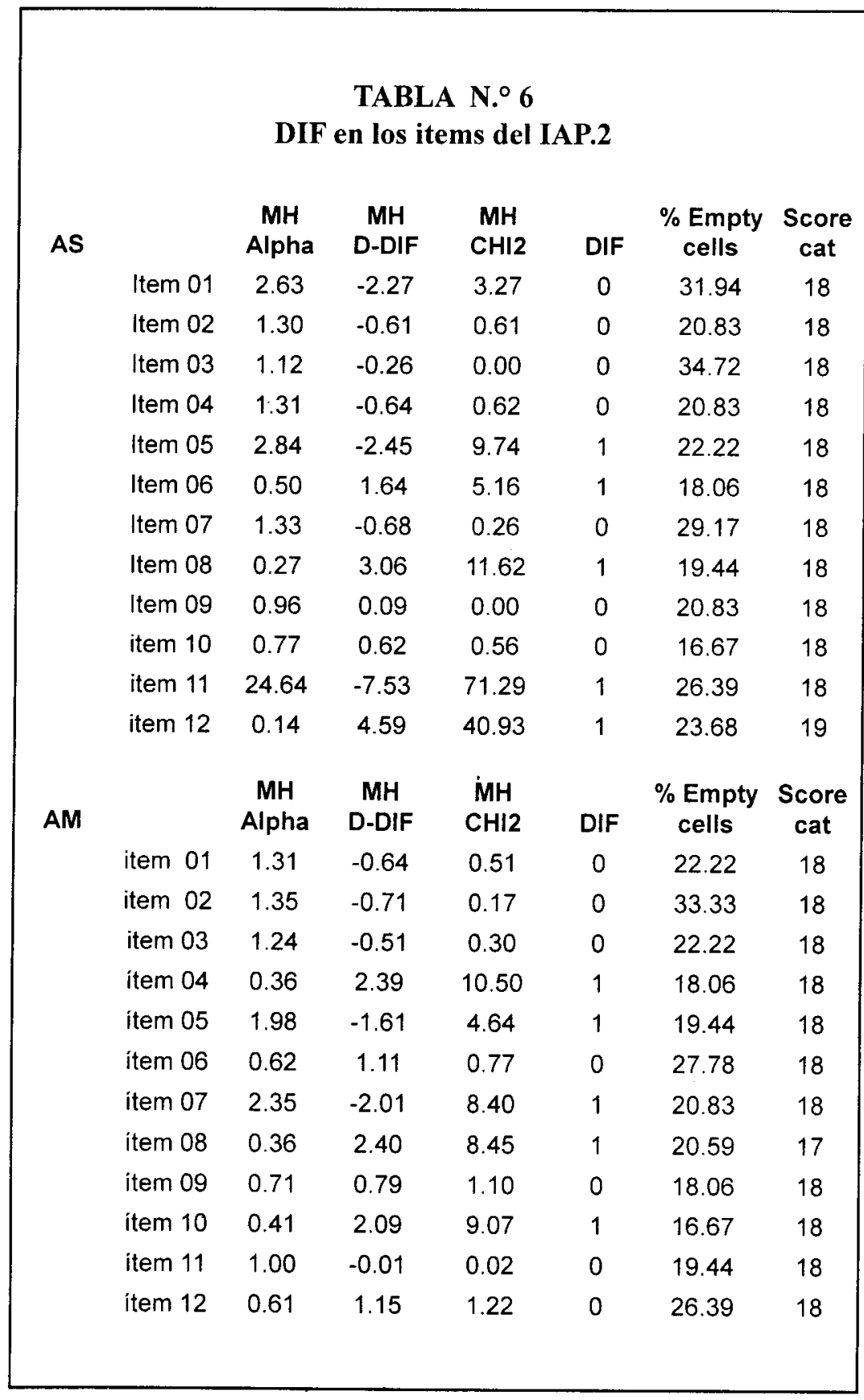


TABLA N. ${ }^{\circ} 6$

DIF en los items del IAP. 2 [continuación]

\begin{tabular}{|c|c|c|c|c|c|c|c|}
\hline $\mathrm{AE}$ & & $\begin{array}{c}\text { MH } \\
\text { Alpha }\end{array}$ & $\begin{array}{c}\text { MH } \\
\text { D-DIF }\end{array}$ & $\begin{array}{c}\mathrm{MH} \\
\mathrm{CHI} 2\end{array}$ & DIF & $\begin{array}{l}\% \text { Empty } \\
\text { cells }\end{array}$ & $\begin{array}{c}\text { Score } \\
\text { cat }\end{array}$ \\
\hline & item 01 & 0.73 & 0.73 & 0.24 & 0 & 33.33 & 18 \\
\hline & ítem 02 & 0.41 & 2.07 & 7.16 & 1 & 25.00 & 18 \\
\hline & item 03 & 0.69 & 0.88 & 1.44 & 0 & 19.44 & 18 \\
\hline & item 04 & 0.82 & 0.47 & 0.26 & 0 & 23.61 & 18 \\
\hline & item 05 & 0.44 & 1.93 & 3.06 & 0 & 27.78 & 18 \\
\hline & ítem 06 & 1.05 & -0.11 & 0.00 & 0 & 22.22 & 18 \\
\hline & item 07 & 0.48 & 1.75 & 7.87 & 1 & 21.05 & 19 \\
\hline & item 08 & 0.49 & 1.68 & 5.64 & 1 & 25.00 & 19 \\
\hline & ítem 09 & 0.65 & 1.02 & 1.58 & 0 & 20.83 & 18 \\
\hline & item 10 & 0.46 & 1.83 & 5.53 & 1 & 23.61 & 18 \\
\hline & item 11 & 1.95 & -1.56 & 2.93 & 0 & 23.61 & 18 \\
\hline & ítem 12 & 0.97 & 0.07 & 0.00 & 0 & 15.28 & 18 \\
\hline$A C$ & & $\begin{array}{c}\text { MH } \\
\text { Alpha }\end{array}$ & $\begin{array}{c}\text { MH } \\
\text { D-DIF }\end{array}$ & $\begin{array}{c}\mathrm{MH} \\
\mathrm{CHI} 2\end{array}$ & DIF & $\begin{array}{l}\% \text { Empty } \\
\text { cells }\end{array}$ & $\begin{array}{c}\text { Score } \\
\text { cat }\end{array}$ \\
\hline & ítem 01 & 3.58 & -3.00 & 15.21 & 1 & 18.06 & 18 \\
\hline & item 02 & 3.07 & -2.64 & 2.13 & 0 & 37.50 & 18 \\
\hline & item 03 & 4.01 & -3.26 & 4.63 & 1 & 36.11 & 18 \\
\hline & item 04 & 10.35 & -5.49 & 24.47 & 1 & 31.94 & 18 \\
\hline & item 05 & 0.18 & 4.09 & 23.69 & 1 & 22.22 & 18 \\
\hline & item 06 & 10.45 & -5.51 & 32.67 & 1 & 30.56 & 18 \\
\hline & item 07 & 1.53 & -1.01 & 1.84 & 0 & 15.28 & 18 \\
\hline & ítem 08 & 1.48 & -0.92 & 1.66 & 0 & 16.67 & 18 \\
\hline & item 09 & 3.73 & -3.09 & 13.25 & 1 & 27.63 & 19 \\
\hline & item 10 & 0.56 & 1.34 & 3.88 & 1 & 19.44 & 18 \\
\hline & ítem 11 & 0.75 & 0.69 & 0.70 & 0 & 16.67 & 18 \\
\hline & item 12 & 5.63 & -4.06 & 32.90 & 1 & 20.83 & 18 \\
\hline
\end{tabular}




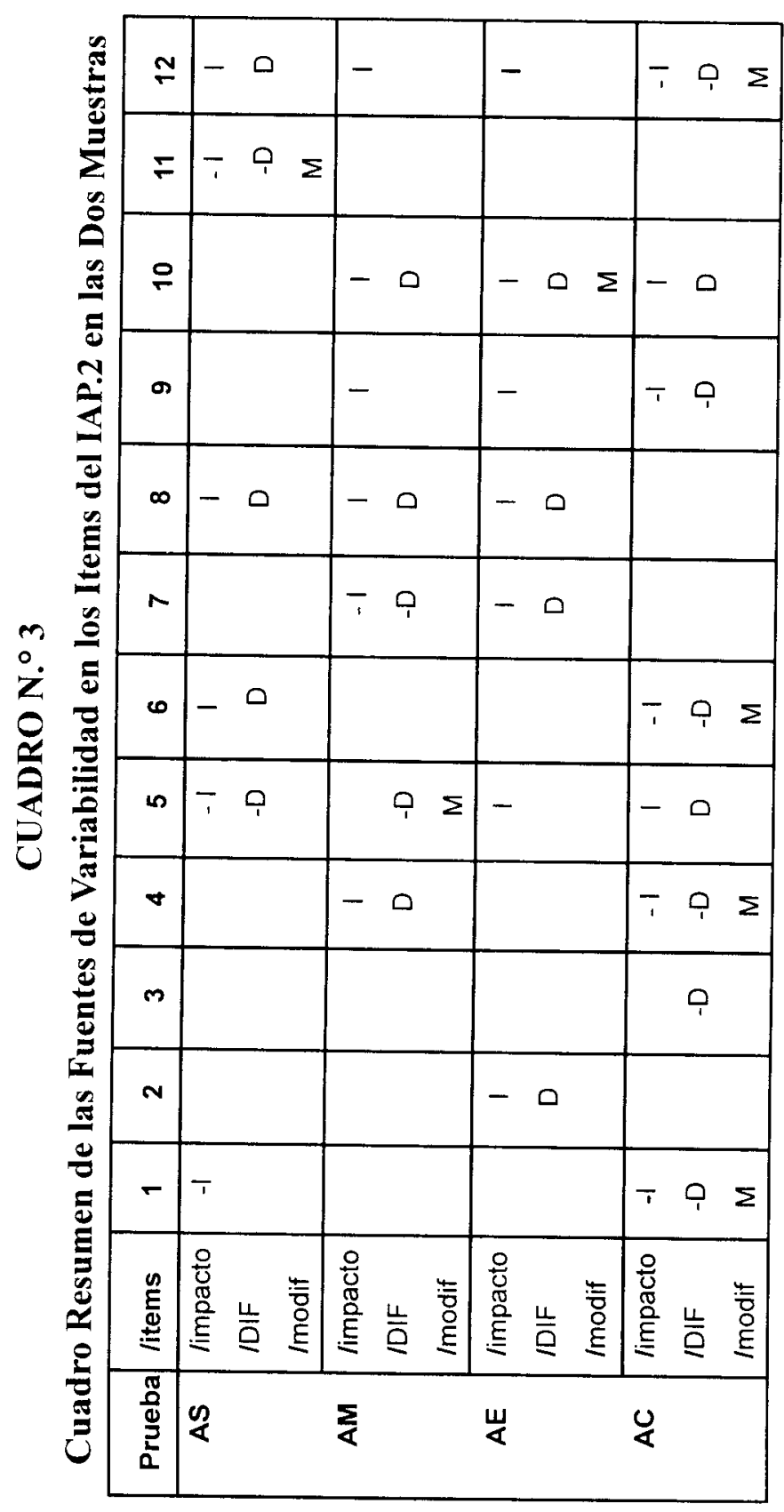


En el cuadro 3 se observan, pues, estos datos:

1. Se han encontrado 23 de los 48 items, que se presentan impacto (I). El sentido del impacto varía de una muestra a otra de este modo. Así está a favor del grupo:

a. español en: $\operatorname{AS}(6,8,12) ; \operatorname{AM}(4,8,9,10,12)$; $\operatorname{AE}(2,5,6,7,8,9,10) ; \mathrm{AC}(5,10)$

b. dominicano en: $\operatorname{AS}(1,5,11,12)$; $\operatorname{AM}(7)$; $\mathrm{AE}(0) ; \mathrm{AC}(1,4,6,9,12)$

2. El DIF aparece en 21 de los 48 items. El sentido del DIF varía, así mismo, de una muestra a otra. Así está a favor del grupo:

a. español en: AS $(6,8,12)$; AM $(4,8,10)$; $\operatorname{AE}(2,7,8,10) ; \mathrm{AC}(5,10)$

b. dominicano en : $\mathrm{AS}(5,11)$; $\mathrm{AM}(5,7) ; \mathrm{AE}(0)$; $\mathrm{AC}(1,3,4,6,9,12)$

3. De los 7 items, que fueron modificados (M)por razones culturales, se observa que:

a. 6 presentan impacto a favor del grupo dominicano (AS-11 AE-10, AC-1, AC-4, AC-6, AC-12)

b. y 1 tan sólo, presenta DIF a favor del grupo dominicano (AM-5)

Para facilitar la discusión del los datos del Cuadro N. ${ }^{\circ}$, se han organizado de nuevo los datos del Cuadro $\mathrm{N}^{\circ} 4$, agrupando los items en los procesos mentales propuestos, indicando debajo de cada item la presencia y sentido del: Impacto (I), DIf (D) y Modificación (M).

\section{Discusión}

$\mathbf{1}^{\circ}$ La variable independiente sexo no parece influir significativamente $(p<0.05)$ sobre ninguna de las puntuaciones obtenidas ( $\mathrm{AV}, \mathrm{AM}, \mathrm{AE}, \mathrm{AC}, \mathrm{T})$ en el Test 


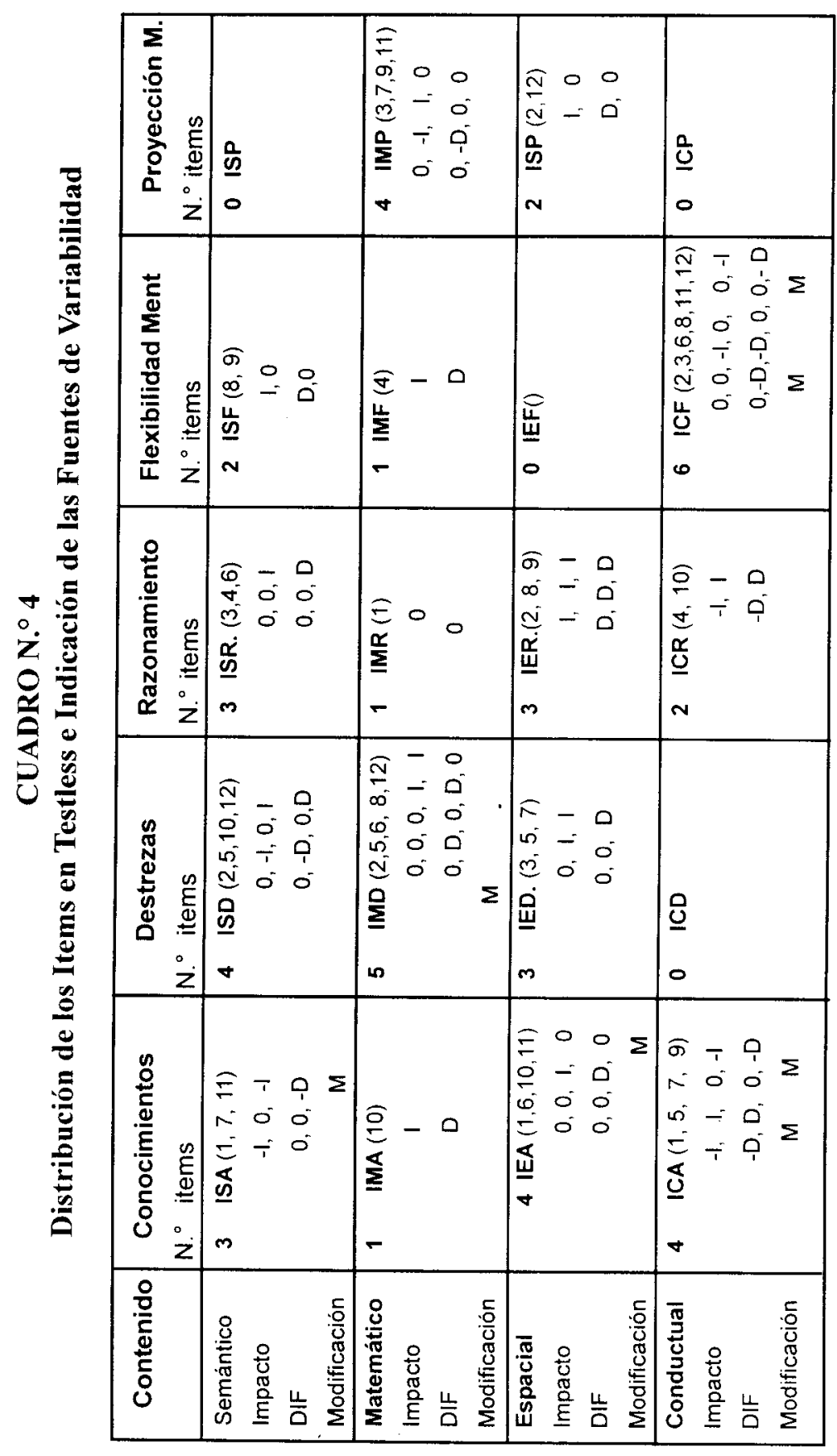


IAP.3, tanto en las dos muestras tomadas por separado, como en la muestra total, ya que las valores $\mathbf{F}$ hallados presentan $\mathbf{p}<0.05$. No obstante se puede observar que, constantemente, en todas las puntuaciones la media de las mujeres es mayor a la de los hombres. Resultado que no es de extraeñar, ya que en los centros seleccionados se imparte una educación mixta y que a ese nivel educativo ( $6^{\circ}$ curso de primaria) las mujeres presentan mayor madurez que los hombres.

$\mathbf{2}^{\mathbf{0}}$ En cambio, la variable edad si parece influir significativamente $(\mathrm{p}<0.05)$ sobre alguna de las puntuaciones del Test IAP.2; así:

- en la muestra dominicana aparecen diferencias en la puntuación AV entre los grupos $1(\mathrm{~N} 1=4)$ vs 2 $(\mathrm{N} 2=86)$ y $1(\mathrm{~N} 1=4)$ vs $3(\mathrm{~N} 2=31)$ a favor del grupo 1 , lo que podría ser explicado por el hecho que el test fue aplicado en el $2^{\circ}$ trimestre del curso 2000 2001, por lo que los resultados pueden estar sesgados por el escaso número de sujetos del grupo 1 , lo que hace pensar que estos alumnos fueron adelantados de curso por su alta madurez mental, madurez que se manifiesta principalmente en los contenidos verbales.

- en cambio en la muestra española la diferencia aparece sólo en las puntuaciones AM entre los grupos $2(\mathrm{~N} 2=112)$ y $3(\mathrm{~N} 3=21)$ a favor del grupo 2 , lo que podría ser explicado por el hecho que el test fue aplicado en el $1^{\circ}$ trimestre del curso 2000-2001, por lo que el grupo 3 puede estar formado por un número importante de alumnos con cierto retraso escolar.

$3^{0}$ Respecto a la variable centro sí parece influir significativamente $(\mathrm{p}<0.05)$ sobre casi todas las 
puntuaciones del Test IAP.2; así: en la puntuación AM aparece una diferencia significativa $(p=0.0122)$ entre las dos muestras ( 1 española, 2 dominicana) a favor de la muestra española, así mismo están diferencias se muestran muy significativas en la puntuación $\mathrm{AE}$ a favor de la muestra española $(p=0.0001)$ y en la puntuación $\mathrm{AC}$ a favor de la muestra dominicana $(p=0.00001)$. No aparecen diferencias significativas en las puntuaciones $\mathrm{AV}$ y ni en la Total. Estos resultados pueden inducir a exponer algunas reflexiones generales sobre los sistemas educativos de cada país, tales como que el sistema educativo español tiende a dar mejor formación que el dominicano en el procesamiento mental de los contenidos matemáticos y espaciales, en cambio el sistema educativo dominicano está desarrollando la llamada inteligencia práctica (Wagner, 1994)

$4^{\circ}$ Finalmente, la variable país sí parece influir significativamente $(\mathrm{p}<0.05)$ sobre casi todas las puntuaciones del Test IAP.3; asi atendiendo a:

los contenidos se tiene que:

- en la puntuación AM aparece una diferencia significativa $(\mathrm{p}=0.0122)$ entre las dos muestras (1 española, 2 dominicana) a favor de la muestra española,

- así mismo estas diferencias se muestran muy significativas en la puntuación $\mathrm{AE}$ a favor de la muestra española $(\mathrm{p}=0.0001)$,

- en cambio en la puntuación AC la diferencia está a favor de la muestra dominicana $(p=0.00001)$,

- no aparecen diferencias significativas en las puntuaciones $\mathrm{AV}$ y ni en la Total. 
Estos resultados pueden inducir a exponer algunas reflexiones generales sobre los sistemas educativos de cada país, tales como que el sistema educativo español tiende a dar mejor formación que el dominicano en el procesamiento mental de los contenidos matemáticos y espaciales, en cambio el sistema educativo dominicano tiende desarrollar la llamada inteligencia práctica (Wagner, 1994)

\section{Los procesos mentales}

Dado que el IAP. 3 ha sido construido de acuerdo a un modelo de procesamiento cognitivo, es posible estudiar mejor estas diferencias entre los sistemas educativos si se efectúa un análisis sustantivo de los items de cada una de las pruebas, agrupándo los items en pequeños tests (testless) que miden procesos:

TABLA N. ${ }^{\circ} 9$

Análisis sustantivo de los items, en el que se indica el número de items que presentan $Z$ significativas, sobre total de items de cada proceso o testless.

\begin{tabular}{|c|c|c|c|c|c|}
\hline $\begin{array}{c}\text { Sub- } \\
\text { Pruebas }\end{array}$ & $\begin{array}{c}\text { Procesos: } \\
\text { Conocimientos }\end{array}$ & Destrezas & Razonamiento & Flex Mental & Proy. Mer \\
\hline AV & $\operatorname{ISA}(3 / 4)$ & $\operatorname{ISD}(1 / 3)-$ & $\operatorname{ISR}(1 / 3)+$ & $\operatorname{ISF}(1 / 2)+$ & $\operatorname{ISP}(0 / 0)$ \\
\hline AM & $\operatorname{IMA}(1 / 1)+$ & $\operatorname{IMD}(2 / 4)+$ & $\operatorname{IMR}(0 / 1)$ & $\operatorname{IMF}(1 / 1)+$ & $\operatorname{IMP}(2 / 4)$ \\
\hline$A E$ & $\operatorname{IEA}(1 / 4)+$ & $\operatorname{IED}(2 / 3)+$ & $\operatorname{IER}(3 / 3)+$ & $\operatorname{IEF}(0 / 0)$ & $\operatorname{IEP}(0 / 2)$ \\
\hline$A C$ & $\operatorname{ICA}(4 / 4) \quad-$ & $\operatorname{ICD}(0 / 0)$ & $\operatorname{ICR}(2 / 2)+$ & $\operatorname{ICF}(2 / 6)-$ & $\operatorname{ICP}(0 / 0)$ \\
\hline
\end{tabular}

(") Los signos (+ -) indican el sentido de la diferencia: + a favor de la muestra española; - a favor de la muestra dominicana

Los datos de la TABLA N. 9 permiten hacer las siguiente inferencias especificas referentes a los procesos mentales analizados:

$\mathbf{1}^{\mathbf{0}}$ Se ha visto antes que no aparece una diferencia significativa entre las muestras de los dos paises en las puntuaciones globales de la subprueba AV. No obstante, 
en el análisis sustantivo de los items se encuentran items, que si lo hacen ( 3 de 4 ); esta diferencia favorece en dos de los items de dominio de vocabulario (ISA) a los dominicanos y uno a los españoles; la diferencia en el dominio de las destrezas lingüísticas (ISD) se da en un solo ítem, que favorece a los dominicanos, sin embargo en los procesos de razonamiento y flexibilidad mental la diferencia favorece a la muestra española. Es decir que la ventaja va de un lado al otro de las muestras. Ello permite señalar que la diferencia parece favorecer a los dominicanos en los procesos más cargados de aprendizaje -inteligencia cristalizada-, en cambio favorece a los españoles en los prcesos que implican la utilización de las aptitudes mentales innatas -inteligencia fluída- (Allik \& Realo, 1997; Lee, Wong \& Alt, 2000).

$2^{\mathbf{0}}$ Anteriormente se ha visto que aparece una diferencia significativa en las puntuaciones globales de la subprueba AM, entre las muestras de los dos países. Al realizar el análisis sustantivo de los items se encuentra que estas diferencias favorecen en la mayoría de los casos a la muestra española, excepción hecha de un 1 de los 4 items referentes a la proyección mental (IMP). Esto induce a pensar que la preparación académica en los contenidos matemáticos de los españoles se evidencia superior a la de los dominicanos, sobre todo en el uso de algunos contenidos, tales como: cálculo mental, conocimiento del sistema métrico decimal, operaciones con quebrados... En cambio en la aplicación del sentido práctico (ítem 7 ) el dominicano es significativamente mejor.

$3^{\circ}$ Así mismo se ha visto que aparece una diferencia significativa en las puntuaciones globales de la subprueba $\mathbf{A E}$, entre las muestras de los dos países. Al 
realizar el análisis sustantivo de los items se encuentra que estas diferencias favorecen en la mayoría de los casos a la muestra española, así en uno de los cuatro items de dominio de conocimientos estructurales (IEA) la diferencia está a favor de los españoles. Esta diferencia se da, también, en dos de los tres items referentes al dominio de las destrezas espaciales (IED: rotación, simetría,...), sin embargo esta diferencia se evidencia más en los procesos de razonamiento (IER) (tres de los tres items existentes). No aparece ningún item de los dos items referentes a la proyección mental. En general se confirma lo visto anteriormente que la diferencia significativa favorece a los españoles en la utilización de las aptitudes innatas (inteligencia fluida).

$4^{\mathbf{0}}$ Sin embargo, se ha visto que aparece una alta diferencia significativa a favor de los dominicanos en las puntuaciones globales de la subprueba $\mathbf{A C}$. Al realizar el análisis sustantivo de los items se encuentra que estas diferencias favorecen en todos los casos a la muestra dominicana. Así esta diferencia favorece a los dominicanos en cuatro de los cuatro items de dominio de conocimientos referentes al sentido social (ICA). Esta diferencia se da, también, en dos de los dos items referentes a los procesos de razonamiento (ICR), así como en dos de los seis items referentes a flexibilidad mental (ICF). No aparecen items en los testless ICD e ICP.

Los resultados obtenidos, confirman plenamente, la hipótesis inferida consistentemente en las anteriores inferencias, que el sistema educativo dominicano tiende a favorecer el desarrollo de la inteligencia práctica y conocimientos aprendidos (inteligencia cristalizada), en cambio el sistema educativo español tiende a favorecer el desarollo de las potencialidades de los sujetos o inteligencia fluida. 


\section{REFERENCIAS BIBLIOGRÁFICAS}

Allik, J y Realo, A. (1997). Intelligence, academic abilities and persolaty. Personality and Individual Defferences. Vol. 23, 809-814.

Anderson, J.R. (1983). The architecture of cognition. Cambridge, MA: Harvard University Press.

Anderson, J.R. (1993). Rules od the mind. Hillsdale, NJ: Lawrence Eribaum Associtates Inc.

Baker, H.J. y Leland, B. (1967). Detroit Test of Learning Aptitude. Indianapolis: Bobbs-Merrill.

Binet, A. (1911). Nouvelles Recherches sur la measure du niveau intellectuel chez les enfants d'echole. Revue Philosophie, 11, 191-244.

Binet, A. y Simon, T. (1905). Methodes nouvelles pour le diagnostique du niveau intellectuel des anormaux. Année Psychologique, 11, 191-244.

Binet, A. y Simon, T. (1908). Le developpement de l'intelligence chez les enfants. Anné Psychologique, 14, 1-94

Broom, M.E. (1930). A further study of validity of test of social intelligence. Journal of Educational Research, 22, 403-405.

Carroll, J.B (1993). Test Theory and Behavioral Scaling of Test Performance. (pp. 276-297). In N. Frederiksen, R.J. Mislevy y I.I. Bejar (Eds.). Test Theory for a New Generation of Tests. (pp. 269-322). Hillsdale, NJ: Lawrence Erlbaum Associates.

Carter, H.D. (1959). Improving the prediction of school achievement by the use of the California Study Methods Survey. Education Administraion and Supervision, 45, 255-260.

Catell, R.B. (1963). Theory of fluid and crystallized intelligence: A Critical experiment..Journal of Educational Psychology Measurement, 54,, 122.

Catell, R.B. (1971). Abilities: Their structure, growth, and action. New York, NY: Houghton Mifflin.

Catell, R.B. (1978). The scientific use of factor. New York, NY: Plenum Press.

Das, J.P. y Naglieri, J.A. (1987,1996). Cognitive Assessment System.

Deriaz, W. (1929). Deux types d'intelligence. Archives de Psicologie, 22,115.

Detroit Test of Learnong Aptitute-DTLA- (1967, 1985, 1991), 
Díaz, J. V. (2000). Introducción a la Métodos de Investigación y Medición Psicológicos: Construcción de cuestionarios, escalas y tests. Valencia, Ed. Cristóbal Serrano.

Díaz, J.V. (2001): Hacia la evaluación de la Inteligencia Académica y del Rendimiento Escolar. Ciencia y Sociedad, Vol. XXVI, 2, 151-203 (RD ISSN.0378-7680)

Diaz, J.V. (2001): Procediminetos básicos para la construcción de tests de aptitud y rendimiento. Material de apoyo al curso del mismo título impartido en la Universidad Nacional Pedro Henriquez Ureña, Sto Domingo, RD (febrero-marzo 2001). En prensa, 141 pgs

Elliot, C.D. (1990). Deferential Ability Scales (DAS). San Antonio, TX: Psychological Corporetion,

Ford, M.E. y Tisak, M.S. (1983). A Further Search for Social Intelligence. Journal of Educcational Psychology, v75, n2, 196-206.

Gardner, H. (1983). Frame of mind: The Theory of multiple intelligences. New York: Basic Books.

Gough, H.G. (1948). A note on the Security- Insecurity test. Journal of Social Psychology, v28, 257-261.

Gough, H.G. y Weiss, D.S. (1981). A notransformational test of intellectual comptence. Journal of Applied Psychology, v66(1), 102-110.

Guilford, J.P., Hoepfner, R. y Petersen, Hugh (1965). Predicting achievement in ninth-grade mathematics from measures of intellectual aptitude factors. Educational and Psychological Measurement. Vol. 25(3), 659-682.

Guilford, J. P. y Hoepfner, R. (1966). Structure-of-Intellect Tests and Factors.Reports from the Psychological Laboratory, University of Southern California, No 36.

Guilford, J. P. (1967). The nature of human intelligence. New York: McGraw-Hill.

Holowinsky, I. (1961). The relationship betewn intelligence (80-110 I.Q.) and achievment in basic eduational skills. Trainning Schooll Bulletin, $58,14-22$.

Hammill, D.D. (1985). Detroit Tests of Learning Aptitude. (DTL-2). Austin, TX pro. ed.

Hammill, D.D. (1991). Detroit Tests of Learning Aptitude. (DTL-3) Austin, TX pro. ed. 
Horn, J.L. (1968). Organization of abilities and development of intelligence. Psychological Review, 75, 242-259.

Jensen, A. R. (1993). Test validity: g versus "tacit knowledge". Current Directions in Psychological Science, Vol. 2(1), 9-10.

Jensen, A.R. (1979). g, outmoded or unconquered frontier?Creative Science and Technology, $2,16-29$.

Jones, K. y Day, J.D. (1996). Cognitive betewn acdemically and socially giftes studens. Roeper Review. Vol. 18, 270-273.

Jones, K. y Day, I.D. (1997). Discrimination of two aspects of cognitivesocial intelligence from academic intelligence. Personality and Individual Defferences. Vol. 89, 486-497.

Kaufman,A.S. y GKaufman, N.L. (1983). Kuafman Assessment for Children. Circle Pines, MN: Amican Guidence Service.

Kaufman,A.S. y GKaufman, N.L. (1993). Kuafman Adolescence and Adult Intelligence Test. Circle Pines, MN: Amican Guidence Service.

Keating, D.P. (1978). A search for social intelligence. Journal of Educational Psycn 'ogy, Vol. 70(2), 218-223.

Kovarsky, V. (1933). Inspection psycholgique scolaire. Monpellier, Public Scolls.

Kolowinsky, I. (1961). The relationship between intelligence (80- 110 I.Q.) and achievement in basic educational.skills. Training School Bulletin, $58,14-22$.

Lee, J.E., Wong, Ch.M.T., Day, J.D., Maxwell, S.E. y Thorpe, P. (2000). Social and academic intelligence: A mut-trait-mildiensional study of crystallized and fluid charactrísitics. Personality and Individual Diferences, vol. 29(3), 539-533.

LXR. Test 5.1 (1995). Logic eXtension Resources. Rancho Cucamonga, CA.

McBee, G y Duke, R.L. (1960). Relationship betwen intelligence, scholastic motivation, an academic achievement. Psychological Reports, 6, 3-8.

McGrew, K.S y Flanagan, D.P. (1996). The intelligence test desk reference (ITDR): Gf-GC croos battery assessment. Boston, MA: Allyn and Bacon.

Mierke, K (1938). Ueber die "practike Veralangung"/ Concerning "practical aptitude". Zeitschrift fuer Angewante Psychologie, 55, 154-192. 
Oliver, R.N, (1994). A correlational study of children's social intelligence, social influence, and academic achievement. Dissertation Abstract Internaional: Section A: Humanities and Social Sciences, Vol 55 (3A): 467.

Piaget, J. (1955). Th psychology of Intelligence. Totowa, NJ: Littlefield Admas.

Piaget, J. (1972). Th psychology of Intelligence. Totowa, NJ: Littlefield Admas.

Riggio, R.E., Messamer, J y Throckmorton, B.(1991) Social and academic Intelligence: Conceptually distinct but overlapping constructs. Personality and Individual Diferences, vol. 12(7), 695-702.

Rusu, L. (1932). Aptitudinea tehnica si inteligencia practica/Technical ability and practical intelligence. Cluj, Rumania, Inst. De Psihol. Unv. Cluj, viii, $141 \mathrm{pp}$

Sánchez-Cánovas J y Sánchez López, M.P (1994). Psicología diferencial: diversidad e individualidad humanas. Madrid. Ed. Centro de Estudios Ramón Areces.

Scudder, C.R. y Raubenheimer, A.S. (1930). Are standarized mechanical aptitude tests valid? Journal of Juvenile Research, 14, 120-123.

Scheringer, E. (1928). Experimental studies of perceptual-motor combination (practical intelligence).Langensalza, Bayer and Son.

Secadas, F. (1995). Inteligencia y Cognición. Rev de Psicología Aplicada, $48(4), 511-537$.

Sechrest, L., Jackson, D.N (1961). Social intelligence and accuracy of impersonal predictions. Journal of Personality, Vol. 29, 167-182.

Shukle, N.N. (1958). Relation of intelligence and ability to scholastic achievement of pupils in S.S.C. class. Journal of Vocational and Educational Guidence. Vol. 5, 38-42.

Singh, B. (1956). Correlation between intelligence and schools achievements. Vidya Bhawan Studies, 5: 1-15.

Spearman, C. (1904). "General Intelligence" objectively determined and measured Amer.J.Psychol.15, 201-292

Spearman, C. (1907). Demonstration of formulate for true measurements of correlation. Amer. J.Psychol.18, 161-169.

Spearman, C. (1910). Correlations calculated from faulty data. Britsh Journal Psychology, 3, 271-295. 
Spearman, C. (1913). Correlations of sum and differences. Britsh Journat Psychology, 5, 417-426.

Spearman, C. (1927). The abilities of man. New York: Macmillan.

Stern, W. (1914). The Psychological Methods of Testing Intelligence. Educational Psychol. Monograph. No 13. Baltimore: Warwick and York.

Sternberg, R. J. (1996). IQ Counts, but What Really Counts is Successful Inteligence. NASSP Bulletin, v 80. n 583, p 18-23.

Sternberg, R. J., Conway, B.E., Ketron, J.L., y Bernstein, M. (1981). Peopel's conception Intelligence. Journal of Personality and Social Psychologv. Vol. 41(1), 37-55

Sternberg, R. J., Forsythe, G.B., Hedlund, J., Horvath, J.A., Wagner. R.K., Williams, W.M., Snook, Scott A., Grigorenko, E.L. (2000). Practice Intelligence everyáay. New York, Cambridge University Pres.

Stewart, J.H. (1998). Practice Intelligence: Assessing its convergent and discriminant validity with social, emocional and academic Intelligence. Dissertation Abstract Internaional: Section B: The Sciences and Engineering, Vol 58 (8-B): 4504.

Terman, L.M. \& Merril, M.A (1937). Measurement of Inteligence. Boston: Houghton Mifflin.

Terman, L.M. \& Merril, M.A (1960).Stanford-Binet Intelligence Scale: mamual for the Terman, L.M.(1916). The measurement of intelligence. Boston: Mifflin. Houghton,

Thorndike, E.L. (1907). An introduction to theory of mental and social measurement. New York. Science Press.

Thorndike, E.L. (1922). On finding equivalent scores in tests of intelligence J. Appl. Psychol. 6,29-33.

Thorndike, E.L. et alt (1927). The measurement of Intelligence. . New York: Bureau of Publications, Teachers College, Columbia University. Pp. $x x v i+616$

Thurstone, L.L. (1929). Theory of attitude measurement. Psychological Review. Vol.36, 222-241.

Thurstone, L.L. (1935). The vectors of mind. Chicago: University of Chicago Press.

Thurstone, L.L. y Thurstone, T.G. (1941). Factor Studies of Intelligence Psychometric Monogr. 2. Chicago: University of Chicago Press. 
Thurstone, L.L.(1938). Primary mental abilities. Chicago: University of Chicago Press.

Torf B. y Sternberg, R.J.(1998). Changing mind, changing world: Practical intelligence and tacit knowledge in adult learning. In M.Cecil Smith y Thomas Pourchot (Eds): Adult Learning and development: Perspectives from educational psychology. The Educational Series (pp.109-126) Mahwah, NJ: Lawrence Erlbuam Associates, Inc. Publishers

Tsudsuki, A. (1957). Prediction of the scolastic achievement in middleschool pupils. Bulletin of the Faculty of Edrcation-L-Nagoya, Vol. 3. 394.

Vernón, R.E. (1971). The structure of human abilities. London: Methuen.

Wagner, R. K (1994). Practical intelligence. European Journal of Psychological Assesment, Vol. 10(2), 162-169.

Wagner, R.K y Kistner, J.A. (1990). Implications of distintion betewn academic and practical intelligence for learning-disabled children. En H.L. Swanson, B.K. Keogh \& Alt (Eds): Leurning desabilities: theoretical and research issues (pp. 75-92). Hillsdale, NJ: Lawrence Erlbaum Associates, Inc.

Warhadpande, N.R. y Kuller, M.P (1966). Schollastic abilities of pupils of Nootan Marathi Vidyalaya. Manas, 13(1), 11-28.

Weschler, D. (1939). The measurement of Adult Inteligence. Baltimore: Williams \& Wilkins.

Weschler, D. (1941). The measurement of Adult Intelligence. Baltimore: Williams \&Wilkins, .

Weschler, D. (1944). The measurement of Adult Intelligence. Baltimore: Williams \& Wilkins,.

Weschler, D. (1955). Manual of the Weschler Adult Intelligence Scale. New York: Psychological Corporation.

Weschler, D.(1967).Manual of the Weschler Preschool and Primary Scale Intelligence. New York: Psychological Corporation

Weschler,D.(1949). Manual of the Weschler Intelligence Scale for Children. New York: Psychological Corporation.

Williamson, E.G. y Darley, J.G (1935). Matching abilities to jobs. Personnel Journal, 13, 344-352.

Wong, Ch.M, Day, J.D., Mexwell, S.E. y Meara, N,M, (1995). A multitraitmultimethod study of academic and social in college students. Journal of Educational Psychology, 87(1), 117-133. 
Yamamoto, K. (1964). Threshold of intelligence in academic achievement of highly creative students. Journal of Experimental Education. Vol.

24(4), 401-405. 